\title{
An Inequality for Tutte Polynomials
}

\author{
Bill Jackson*
}

8 February 2008

\begin{abstract}
Let $G$ be a graph without loops or bridges and $a, b$ be positive real numbers with $b \geq a(a+2)$. We show that the Tutte polynomial of $G$ satisfies the inequality $T_{G}(b, 0) T_{G}(0, b) \geq T_{G}(a, a)$. Our result was inspired by a conjecture of Merino and Welsh that $T_{G}(1,1) \leq$ $\max \left\{T_{G}(2,0), T_{G}(0,2)\right\}$.
\end{abstract}

\section{Introduction}

The Tutte polynomial, or dichromate, of a graph $G=(V, E)$ was discovered by Tutte [7]. It has wide ranging applications from knot theory to statistical mechanics. The polynomial can be defined by the closed formula

$$
T_{G}(x, y)=\sum_{A \subseteq E}(x-1)^{r(E)-r(A)}(y-1)^{|A|-r(A)}
$$

where $r(A)=|V|-\omega(V, A)$, and $\omega(V, A)$ denotes the number of components in the graph $(V, A)$. It can also be calculated recursively by putting $T_{G}(x, y) \equiv 1$ when $E(G)=\emptyset$, and using the following fundamental lemma.

Lemma 1 [7] Let $G$ be a graph and $e \in E(G)$. Then

(a) $T_{G}(x, y)=T_{G-e}(x, y)+T_{G / e}(x, y)$ if $e$ is neither a loop not a bridge,

(b) $T_{G}(x, y)=x T_{G-e}(x, y)$ if e is a bridge,

(c) $T_{G}(x, y)=y T_{G-e}(x, y)$ if $e$ is a loop.

(d) Furthermore, if $G=G_{1} \cup G_{2}$, where $E\left(G_{1}\right) \cap E\left(G_{2}\right)=\emptyset$ and $\mid V\left(G_{1}\right) \cap$ $V\left(G_{2}\right) \mid \leq 1$, then $T_{G}(x, y)=T_{G_{1}}(x, y) T_{G_{2}}(x, y)$.

* School of Mathematical Sciences, Queen Mary, University of London, Mile End Road, London E1 4NS, England. e-mail: b.jackson@qmul.ac.uk. 
A proof of this lemma together with further information on Tutte polynomials can also be found in the text book by Tutte [8], and in the survey article of Brylawski and Oxley [1]. We may use Lemma 1 and induction to deduce that the Tutte polynomial of a graph with at least one edge has nonnegative coefficients and zero constant term.

The Tutte polynomial of a graph contains a large amount of information about the graph. It follows from equation (1) that $T_{G}(1,1)$ is equal to the number of spanning trees of a connected graph $G$. Stanley [6] showed that $T_{G}(2,0)$ is the number of acyclic orientations of $G$. It also follows from either Greene and Zaslavsky [3] or Las Vergnas [4], see [1, Example 6.3.8], that $T_{G}(0,2)$ is the number of totally cyclic orientations of $G$ i.e. orientations in which every arc lies in a directed cycle. Merino and Welsh [5] conjectured that the following intriguing inequality holds between these three evaluations of the Tutte polynomial.

Conjecture 2 For all 2-connected graphs $G$,

$$
T_{G}(1,1) \leq \max \left\{T_{G}(2,0), T_{G}(0,2)\right\} .
$$

Some evidence in favour of the conjecture has recently been given by Conde and Merino in [2]. They also state the following stronger conjecture.

Conjecture 3 For all 2-connected graphs $G$,

$$
T_{G}(2,0) T_{G}(0,2) \geq T_{G}(1,1)^{2} .
$$

Note that Conjectures 2 and 3 become false if we allow loops or bridges. If $G$ has a loop then $T_{G}(2,0)=0$ and if $G$ has a bridge then $T_{G}(0,2)=0$. Since $T_{G}(1,1)$ can be arbitrarily large in both cases, Conjecture 3 would not hold. To see that Conjecture 2 becomes false if we allow loops or bridges but not both, consider the graph $G$ obtained from a circuit of length $m$ by adding a loop. We have $T_{G}(x, y)=y\left(x^{m-1}+x^{m-2}+\ldots+x+y\right)$. Thus $T_{G}(2,0)=0, T_{G}(0,2)=4$ and $T_{G}(1,1)=m$. So for $m \geq 5, T_{G}(1,1)>$ $\max \left\{T_{G}(2,0), T_{G}(0,2)\right\}$. On the other hand, we may use Lemma $1(\mathrm{~d})$ to deduce that Conjecture 3 is equivalent to the apparently stronger conjecture that $T_{G}(2,0) T_{G}(0,2) \geq T_{G}(1,1)^{2}$ for all loopless bridgeless graphs $G$.

The main purpose of this paper is prove the following result which runs in the same vein as Conjecture 3.

Theorem 4 Suppose $G$ is a graph without loops and bridges, and $a \geq 0$, $b \geq a(a+2)$ are real numbers. Then

$$
T_{G}(b, 0) T_{G}(0, b) \geq T_{G}(a, a)^{2} .
$$


Putting $a=1$ and $b=3$ we may deduce that $T_{G}(3,0) T_{G}(0,3) \geq T_{G}(1,1)^{2}$ for all graphs $G$ without loops and bridges.

Our proof of Theorem 4 is given in Section 3. It is an inductive proof based on Lemma 1. The main difficulty in the proof is to stay within the family of loopless bridgeless graphs. We accomplish this by using parallel and series reductions which are described in Section 2.

\section{Parallel and Series Reductions}

We may define an equivalence relation on the edges of a graph $G$ by saying that two edges $e, f \in E(G)$ are equivalent if either $e=f$ or $\{e, f\}$ induces a circuit in $G$. We denote the equivalence class of $e$ by $P_{G}(e)$, or $P(e)$ when it is clear which graph we are referring to, and refer to it as the parallel class of $e$ in $G$. The series class $S_{G}(e)$, or $S(e)$, is defined analogously by saying that $e, f \in E(G)$ are equivalent if either $e=f$ or $\{e, f\}$ induces a cocircuit in $G$ i.e. $\{e, f\}$ forms an edge-cut in $G$. We say that $P(e)$ is a multiple bridge in $G$ if $e$ is a bridge in $G-(P(e)-e)$, and that $S(e)$ is a subdivided loop in $G$ if $e$ is a loop in $G /(S(e)-e)$. The following lemma extends Lemma 1(a) and can be easily deduced from Lemma 1.

Lemma 5 Let $G$ be a graph and $e \in E(G)$.

(a) Let $P(e)$ be the parallel class of e. If $|P(e)|=p$ and $P(e)$ is not a multiple bridge then

$$
T_{G}(x, y)=T_{G-P(e)}(x, y)+\left(y^{p-1}+y^{p-2}+\ldots+1\right) T_{G / P(e)}(x, y) .
$$

(b) Let $S(e)$ be the series class of e. If $|S(e)|=s$ and $S(e)$ is not a subdivided loop then

$$
T_{G}(x, y)=\left(x^{s-1}+x^{s-2}+\ldots+1\right) T_{G-S(e)}(x, y)+T_{G / S(e)}(x, y) .
$$

We may have to apply Lemma 5 iteratively to a graph which is loopless and bridgeless to eventually arrive at other graphs in the same family. To do this we use the following lemma.

Lemma 6 Let $G$ be a 2-connected graph and $e \in E(G)$.

(a) Suppose $P_{G}(e) \neq E(G)$. Let $P=P_{G}(e)$ and $H=G-(P-e)$. Then $H$ is 2-connected and $P_{H}(e)=\{e\}$. Furthermore, if $S=S_{H}(e)$ and $|S|=s \geq 2$, then $T_{G-P}(x, y)=x^{s-1} T_{H-S}(x, y)$ and

$$
T_{G / P}(x, y)=\left(x^{s-2}+x^{s-3}+\ldots+1\right) T_{H-S}(x, y)+T_{H / S}(x, y) .
$$


(b) Suppose $S_{G}(e) \neq E(G)$. Let $S=S_{G}(e)$ and $H=G /(S-e)$. Then $H$ is 2-connected and $S_{H}(e)=\{e\}$. Furthermore, if $P=P_{H}(e)$ and $|P|=p \geq 2$, then $T_{G / S}(x, y)=x^{p-1} T_{H / P}(x, y)$ and

$$
T_{G-S}(x, y)=T_{H-P}(x, y)+\left(y^{p-2}+y^{p-3}+\ldots+1\right) T_{H / P}(x, y) .
$$

Proof: (a) The fact that $H$ is 2-connected follows from the hypotheses that $G$ is 2-connected, and $P_{G}(e) \neq E(G)$ (and hence $|V(G)| \geq 3$ ). The fact that $P_{H}(e)=\{e\}$ follows immediately from the definitions of $P_{G}(e)$ and $H$. The equality $T_{G-P}(x, y)=x^{s-1} T_{H-S}(x, y)$ follows from Lemma $1(\mathrm{~b})$ using the facts that $H-S=(G-P)-(S-e)$ and each edge in $S-e$ is a bridge of $G-P$. The second equality follows from Lemma $5(\mathrm{~b})$ using the fact that $S-e$ is a series class of $G / P$.

(b) The proof is similar to that of (a).

Let $G$ be a 2-connected graph and $e \in E(G)$. We say that the graph $G-$ $(P(e)-e)$ is the parallel reduction of $G$ at $e$, and that the graph $G /(S(e)-e)$ is the series reduction of $G$ at $e$. When $\left|P_{G}(e)\right| \geq 2$ and $P_{G}(e) \neq E(G)$, we define the parallel/series reduction sequence of $G$ at $e$ to be the sequence of graphs $\left(G=G_{0}, G_{1}, \ldots, G_{k+1}\right)$ such that:

- for all even $i, 0 \leq i \leq k,\left|P_{G_{i}}(e)\right| \geq 2, P_{G_{i}}(e) \neq E\left(G_{i}\right)$, and $G_{i+1}$ is the parallel reduction of $G_{i}$ at $e$;

- for all odd $i, 0 \leq i \leq k,\left|S_{G_{i}}(e)\right| \geq 2, S_{G_{i}}(e) \neq E\left(G_{i}\right)$, and $G_{i+1}$ is the series reduction of $G_{i}$ at $e$;

- either $P_{G_{k+1}}(e)=E\left(G_{k+1}\right)$, or $S_{G_{k+1}}(e)=E\left(G_{k+1}\right)$, or $\left|P_{G_{k+1}}(e)\right|=$ $1=\left|S_{G_{k+1}}(e)\right|$.

An illustration of the parallel/reduction sequence of a graph is given in Figure 1.

When $\left|S_{G}(e)\right| \geq 2$ and $S_{G}(e) \neq E(G)$, the series/parallel reduction sequence of $G$ at $e$ is defined analogously, beginning with the series reduction of $G$ at $e$.

Lemma 7 Let $G$ be a 2-connected graph, $e \in E(G)$ with $\left|P_{G}(e)\right| \geq 2$ and $P_{G}(e) \neq E(G)$. Let $\left(G_{0}, G_{1}, \ldots, G_{k+1}\right)$ be the parallel/series reduction sequence of $G$ at e. Let $\left|P_{G_{i}}(e)\right|=p_{i}$ and $\left|S_{G_{i}}(e)\right|=s_{i}$ for $0 \leq i \leq k+1$. Put $Q_{i}=P_{G_{i}}(e)$ for $i$ even, and $Q_{i}=S_{G_{i}}(e)$ for $i$ odd, $0 \leq i \leq k$. Then $G_{i}$ is 


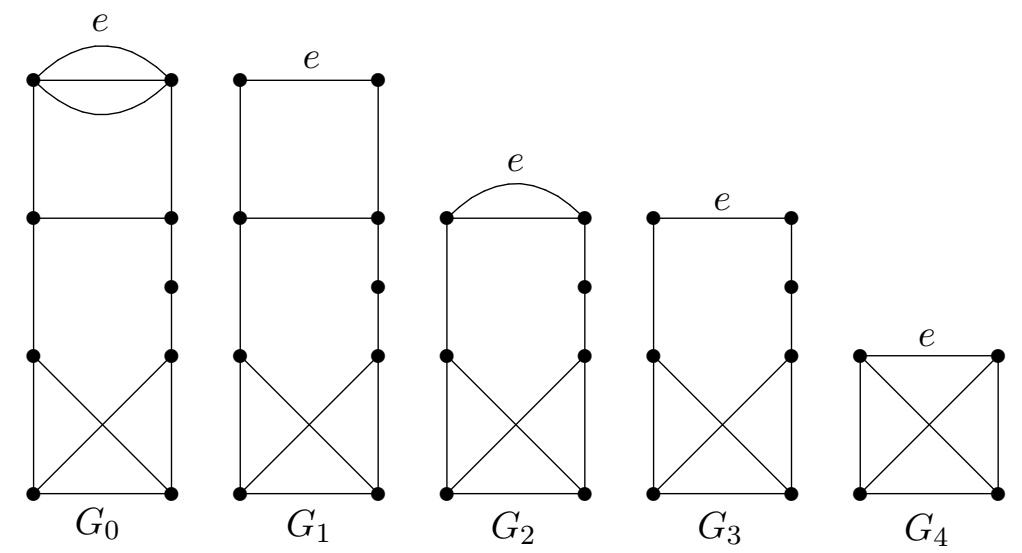

Figure 1: The parallel/series reduction sequence of the graph $G=G_{0}$ at the edge $e$.

2-connected, for all $0 \leq i \leq k+1,\left|S_{i}(e)\right|=1$ for all even $i$, and $\left|P_{i}(e)\right|=1$ for all odd $i$. Furthermore, for all $0 \leq i \leq k$,

$$
T_{G}(x, y)=d_{i}(x, y) T_{G_{i}-Q_{i}}(x, y)+c_{i}(x, y) T_{G_{i} / Q_{i}}(x, y),
$$

where $d_{i}(x, y)$ and $c_{i}(x, y)$ are defined recursively by putting: $d_{0}(x, y)=1$ and $c_{0}(x, y)=y^{p_{0}-1}+y^{p_{0}-2}+\ldots+1 ; d_{i+1}(x, y)=x^{s_{i+1}-1} d_{i}(x, y)+\left(x^{s_{i+1}-2}+\right.$ $\left.x^{s_{i+1}-3}+\ldots+1\right) c_{i}(x, y)$ and $c_{i+1}(x, y)=c_{i}(x, y)$ for all even $i, 0 \leq i \leq k$; $d_{i+1}(x, y)=d_{i}(x, y)$ and $c_{i+1}(x, y)=\left(y^{p_{i+1}-2}+y^{p_{i+1}-3}+\ldots+1\right) d_{i}(x, y)+$ $y^{p_{i+1}-1} c_{i}(x, y)$ for all odd $i, 1 \leq i \leq k$.

Proof: The lemma follows by induction on $i$ using Lemma 5(a) for the base case and Lemma 6 for the inductive step. For example, if $i$ is even then $Q_{i}=P_{G_{i}}(e)=P_{i}$ say, $Q_{i+1}=S_{G_{i+1}}(e)=S_{i}$ say, and $G_{i+1}$ is the parallel reduction of $G_{i}$ at $e$. By induction we have

$$
T_{G}(x, y)=d_{i}(x, y) T_{G_{i}-P_{i}}(x, y)+c_{i}(x, y) T_{G_{i} / P_{i}}(x, y) .
$$

Lemma 6 (a) implies that $T_{G_{i}-P_{i}}(x, y)=x^{s_{i+1}-1} T_{G_{i+1}-S_{i+1}}(x, y)$ and

$T_{G_{i} / P_{i}}(x, y)=\left(x^{s_{i+1}-2}+x^{s_{i+1}-3}+\ldots+1\right) T_{G_{i+1}-S_{i+1}}(x, y)+T_{G_{i+1} / S_{i+1}}(x, y)$.

This gives

$$
T_{G}(x, y)=d_{i+1}(x, y) T_{G_{i+1}-Q_{i+1}}(x, y)+c_{i+1}(x, y) T_{G_{i+1} / Q_{i+1}}(x, y) .
$$


In order to prove our main result we will need the following rather technical lemmas concerning evaluations of the functions $d_{i}(x, y)$ and $c_{i}(x, y)$. We adopt the notation of Lemma 7 , and the convention that an empty product is equal to one. Given $x \in \mathbb{R}$ and a positive integer $t$, put $g(x, t)=$ $x^{t-1}+x^{t-2}+\ldots+1$.

Lemma 8 For all $0 \leq i \leq k$ and all real $a \geq 0$ we have:

(a) $d_{i}(x, 0)=\prod_{j=0}^{i-1} g\left(x, s_{j}\right)$ and $c_{i}(x, 0)=\prod_{j=0}^{i} g\left(x, s_{j}\right)$ when $i$ is even, and $d_{i}(x, 0)=\prod_{j=0}^{i} g\left(x, s_{j}\right)$ and $c_{i}(x, 0)=\prod_{j=0}^{i-1} g\left(x, s_{j}\right)$ when $i$ is odd;

(b) $d_{i}(0, y)=\prod_{j=0}^{i-1} g\left(y, p_{j}\right)$ and $c_{i}(0, y)=\prod_{j=0}^{i} g\left(y, p_{j}\right)$ when $i$ is even, and $d_{i}(0, y)=\prod_{j=0}^{i} g\left(y, p_{j}\right)$ and $c_{i}(0, y)=\prod_{j=0}^{i-1} g\left(y, p_{j}\right)$ when $i$ is odd;

(c) $d_{i}(a, a) \leq \prod_{j=0}^{i-1} g\left(a, s_{j}\right) g\left(a, p_{j}\right)$ and $c_{i}(a, a) \leq \prod_{j=0}^{i} g\left(a, s_{j}\right) g\left(a, p_{j}\right)$ when $i$ is even, and $d_{i}(a, a) \leq \prod_{j=0}^{i} g\left(a, s_{j}\right) g\left(a, p_{j}\right)$ and $c_{i}(a, a) \leq \prod_{j=0}^{i-1} g\left(a, s_{j}\right) g\left(a, p_{j}\right)$ when $i$ is odd.

Proof: The lemma follows from the recursive definition of $d_{i}$ and $c_{i}$ by using induction on $i$ and the facts that $p_{i}=1$ for $i$ odd and $s_{i}=1$ for $i$ even. It is straightforward to check that the base case $i=0$ holds. We give two examples of the inductive step.

Consider statement (a) in the case when $i$ is even. We have

$$
\begin{aligned}
d_{i+1}(x, 0) & =x^{s_{i+1}-1} d_{i}(x, 0)+g\left(x, s_{i+1}-1\right) c_{i}(x, 0) \\
& =x^{s_{i+1}-1} \prod_{j=0}^{i-1} g\left(x, s_{j}\right)+g\left(x, s_{i+1}-1\right) \prod_{j=0}^{i} g\left(x, s_{j}\right) \\
& =\prod_{j=0}^{i+1} g\left(x, s_{j}\right),
\end{aligned}
$$

by induction and the fact that $s_{i}=1\left(\right.$ so $\left.g\left(x, s_{i}\right)=1\right)$. Similarly

$$
c_{i+1}(x, 0)=c_{i}(x, 0)=\prod_{j=0}^{i} g\left(x, s_{j}\right) .
$$

Consider statement (c) in the case when $i$ is odd. We have

$$
d_{i+1}(a, a)=d_{i}(a, a) \leq \prod_{j=0}^{i} g\left(a, s_{j}\right) g\left(a, p_{j}\right),
$$


and

$$
\begin{aligned}
c_{i+1}(a, a) & =g\left(a, p_{i+1}-1\right) d_{i}(a, a)+a^{p_{i+1}-1} c_{i}(a, a) \\
& \leq g\left(a, p_{i+1}-1\right) \prod_{j=0}^{i} g\left(a, s_{j}\right) g\left(a, p_{j}\right)+a^{p_{i+1}-1} \prod_{j=0}^{i-1} g\left(a, s_{j}\right) g\left(a, p_{j}\right) \\
& \leq g\left(a, p_{i+1}\right) \prod_{j=0}^{i} g\left(a, s_{j}\right) g\left(a, p_{j}\right)=\prod_{j=0}^{i+1} g\left(a, s_{j}\right) g\left(a, p_{j}\right),
\end{aligned}
$$

since $g\left(a, p_{i}\right) g\left(a, s_{i}\right) \geq 1$, and $s_{i+1}=1\left(\right.$ so $\left.g\left(a, s_{i+1}\right)=1\right)$.

Lemma 9 For all $0 \leq i \leq k$, and all real $a \geq 0$ and $b \geq a(a+2)$, we have:

(a) $d_{i}(b, 0) d_{i}(0, b) \geq d_{i}(a, a)^{2}$;

(b) $c_{i}(b, 0) c_{i}(0, b) \geq c_{i}(a, a)^{2}$.

Proof: We prove (a) in the case when $i$ is even. The other proofs are similar. By Lemma 8 we have

$$
d_{i}(b, 0) d_{i}(0, b)=\prod_{j=0}^{i-1} g\left(b, s_{j}\right) g\left(b, p_{j}\right) \geq \prod_{j=0}^{i-1} g\left(a, s_{j}\right)^{2} g\left(a, p_{j}\right)^{2} \geq d_{i}(a, a)^{2},
$$

since $g(b, t+1) \geq g(a, t+1)^{2}$ for all nonnegative integers $t$. The latter statement follows since

$$
\begin{aligned}
g(b, t+1) & \geq a^{t}(a+2)^{t}+a^{t-1}(a+2)^{t-1}+\ldots+1 \\
& \geq a^{t}(a+2)^{t}+\sum_{i=0}^{t-1} a^{i} 2^{i} \\
& \geq \sum_{i=0}^{t} a^{2 t-i} 2^{i}+\sum_{i=0}^{t-1} a^{i} 2^{i} \\
& \geq \sum_{i=0}^{t}(i+1) a^{2 t-i}+\sum_{i=0}^{t-1}(i+1) a^{i} \\
& =g(a, t+1)^{2} .
\end{aligned}
$$




\section{Proof of Theorem 4}

We proceed by contradiction. We assume the theorem is false and choose a counterexample $(G, a, b)$ such that $|E(G)|$ is as small as possible. If $E(G)=\emptyset$ then $T_{G}(x, y) \equiv 1$ and the theorem holds for $G$. Thus $E(G) \neq \emptyset$. Since $T_{G}(x, y) \geq 0$ for $x, y \geq 0$, we have $a>0$.

Claim $10 G$ is 2-connected.

Proof: Suppose $G$ is separable. Then there exist two loopless and bridgeless proper subgraphs $G_{1}, G_{2}$ of $G$ such that $T_{G}(x, y)=T_{G_{1}}(x, y) T_{G_{2}}(x, y)$. By the minimality of $G$, the theorem holds for $G_{1}$ and $G_{2}$. Lemma $1(\mathrm{~d})$ now implies that the theorem holds for $G$.

Claim 11 (a) There exists a real number $z_{0}$ such that

$$
T_{G}(b, 0)+z_{0}^{2} T_{G}(0, b)<2 z_{0} T_{G}(a, a) .
$$

(b) For all loopless and bridgeless graphs $H$ with $|E(H)|<|E(G)|$ and all real numbers $z_{1}, z_{2}$, we have

$$
z_{1}^{2} T_{H}(b, 0)+z_{2}^{2} T_{H}(0, b) \geq 2 z_{1} z_{2} T_{H}(a, a) .
$$

Proof: (a) Suppose $T_{G}(b, 0)+z T_{G}(0, b)-2 z T_{G}(a, a) \geq 0$ for all $z \in \mathbb{R}$. Then $T_{G}(b, 0)+z^{2} T_{G}(0, b)-2 z T_{G}(a, a)$ has at most one real root. This implies that $T_{G}(a, a)^{2}-T_{G}(b, 0) T_{G}(0, b) \leq 0$ which contradicts the choice of $G$.

(b) The minimality of $G$ implies that $T_{H}(a, a)^{2}-T_{H}(b, 0) T_{H}(0, b) \leq 0$. Thus $T_{H}(b, 0)+z^{2} T_{H}(0, b)-2 z T_{H}(a, a)$ has at most one real root. Since $H$ is bridgeless and $b>0, T_{H}(0, b)>0$. Hence $T_{H}(b, 0)+z^{2} T_{H}(0, b)-$ $2 z T_{H}(a, a) \geq 0$ for all $z \in \mathbb{R}$. Putting $z=z_{2} / z_{1}$ for $z_{1} \neq 0$ we deduce that $z_{1}^{2} T_{H}(b, 0)+z_{2}^{2} T_{H}(0, b) \geq 2 z_{1} z_{2} T_{H}(a, a)$. The latter inequality also holds trivially when $z_{1}=0$.

Claim 12 Let $e \in E(G)$. Then either $G-e$ contains a bridge or $G / e$ contains a loop.

Proof: Suppose not. Then $G-e$ and $G / e$ are both loopless and bridgeless. Furthermore $T_{G}(x, y)=T_{G-e}(x, y)+T_{G / e}(x, y)$. By Claim 11(b),

$$
T_{G-e}(b, 0)+z_{0}^{2} T_{G-e}(0, b) \geq 2 z_{0} T_{G-e}(a, a)^{2}
$$


and

$$
T_{G / e}(b, 0)+z_{0}^{2} T_{G / e}(0, b) \geq 2 z_{0} T_{G / e}(a, a)^{2} .
$$

Adding these two inequalities and using Lemma 1(a) we obtain

$$
T_{G}(b, 0)+z_{0}^{2} T_{G}(0, b) \geq 2 z_{0} T_{G}(a, a)^{2} .
$$

This contradicts Claim 11(a).

It follows from Claim 12 that every edge of $G$ belongs to either a circuit of size two or a cocircuit of size two. No edge can belong to both by Claim 10. Thus, for every edge $e$ of $G$, we have either $P(e)=\{e\}$ and $|S(e)| \geq 2$, or $S(e)=\{e\}$ and $|P(e)| \geq 2$.

Claim 13 For all $e \in E(G), P(e) \neq E(G) \neq S(e)$.

Proof: Suppose $P(e)=E(G)$. Then $T_{G}(x, y)=x+y^{p-1}+y^{p-2}+\ldots+y$, where $p=|P(e)| \geq 2$. Putting $t=p-1$ and $g(b, t)=t^{p-1}+t^{p-2}+\ldots+1$, we have $T_{G}(b, 0)=b, T_{G}(0, b)=g(b, t+1)-1$, and $T_{G}(a, a)=a+g(a, t+1)-1$. We may now use the fact that $g(b, t) \geq g(a, t)^{2}$ (as was shown in the proof of Lemma 9) to deduce that

$$
\begin{aligned}
T_{G}(b, 0) T_{G}(0, b) & =b[g(b, t+1)-1] \\
& =b\left[b^{t}+b^{t-1}+\ldots+b\right] \\
& =b^{2} g(b, t) \\
& \geq 4 a^{2} g(a, t)^{2} \\
& =\left[2 a^{t}+2 a^{t-1}+\ldots+2 a\right]^{2} \\
& \geq\left[a+a^{t}+a^{t-1}+\ldots+a\right]^{2} \\
& =[a+g(a, t+1)-1]^{2}=T_{G}(a, a)^{2}
\end{aligned}
$$

since $b^{2} \geq 4 a^{2}$ for $b \geq a(a+2)$.

If $S(e)=E(G)$ then $T_{G}(x, y)=x^{s-1}+x^{s-2}+\ldots+x+y$, where $s=|S(e)| \geq 2$, and we may proceed similarly; or, more elegantly, we may use that facts that $G$ is planar, $T_{G}(x, y)=T_{G^{*}}(y, x)$ where $G^{*}$ is the planar dual of $G$, and $P_{G^{*}}(e)=E\left(G^{*}\right)$.

Choose $e \in E(G)$. We consider the case when $|P(e)| \geq 2$ and let $\left(G_{0}, G_{1}, \ldots, G_{k+1}\right)$ be the parallel/series reduction sequence of $G$ at $e$. (The case when $|S(e)| \geq 2$ can be treated analogously using the series/parallel reduction sequence of $G$ at $e$.) Let $p_{i}, s_{i}, Q_{i}$ be as defined in Lemma 7 for $0 \leq i \leq k+1$. 
Claim 14 Either $P_{G_{k+1}}(e)=E\left(G_{k+1}\right)$ or $S_{G_{k+1}}(e)=E\left(G_{k+1}\right)$.

Proof: Suppose not. Then we have must $\left|P_{G_{k+1}}(e)\right|=1=\left|S_{G_{k+1}}(e)\right|$ and $P_{G_{k+1}}(e)=\{e\}=S_{G_{k+1}}(e)$. Since $G_{k+1}$ is 2-connected we may deduce that $G_{k}-Q_{k}=G_{k+1}-e$ and $G_{k} / Q_{k}=G_{k+1} / e$ are both loopless and bridgeless. By Lemma 7,

$$
T_{G}(x, y)=d_{k}(x, y) T_{G_{k}-Q_{k}}(x, y)+c_{k}(x, y) T_{G_{k} / Q_{k}}(x, y),
$$

where $d_{k}(x, y)$ and $c_{k}(x, y)$ are as defined in the lemma. We may now use Claim 11(b) and Lemma 9 to deduce that

$$
\begin{aligned}
T_{G}(b, 0)+z_{0}^{2} T_{G}(0, b)= & d_{k}(b, 0) T_{G_{k}-Q_{k}}(b, 0)+c_{k}(b, 0) T_{G_{k} / Q_{k}}(b, 0)+ \\
& z_{0}^{2} d_{k}(0, b) T_{G_{k}-Q_{k}}(0, b)+z_{0}^{2} c_{k}(0, b) T_{G_{k} / Q_{k}}(0, b) \\
= & d_{k}(b, 0) T_{G_{k}-Q_{k}}(b, 0)+z_{0}^{2} d_{k}(0, b) T_{G_{k}-Q_{k}}(b, 0)+ \\
& c_{k}(b, 0) T_{G_{k} / Q_{k}}(b, 0)+z_{0}^{2} c_{k}(0, b) T_{G_{k} / Q_{k}}(0, b) \\
\geq & 2 z_{0}\left[\sqrt{d_{k}(b, 0) d_{k}(0, b)} T_{G_{k}-Q_{k}}(a, a)+\right. \\
& \left.\sqrt{c_{k}(b, 0) c_{k}(0, b)} T_{G_{k} / Q_{k}}(a, a)\right] \\
\geq & 2 z_{0}\left[d_{k}(a, a) T_{G_{k}-Q_{k}}(a, a)+c_{k}(a, a) T_{G_{k} / Q_{k}}(a, a)\right] \\
= & 2 z_{0} T_{G}(a, a) .
\end{aligned}
$$

This contradicts Claim 11(a).

We can now complete the proof of the theorem. We consider the case when $P_{G_{k+1}}(e)=E\left(G_{k+1}\right)$. (The case when $S_{G_{k+1}}(e)=E\left(G_{k+1}\right)$ can be handled similarly.) Since $G_{k+1}$ is 2 -connected we have $p_{k+1} \geq 2$. Hence $k+1$ is even and $Q_{k}=S_{G_{k}}(e)$. By Lemma 7,

$$
T_{G}(x, y)=d_{k}(x, y) T_{G_{k}-Q_{k}}(x, y)+c_{k}(x, y) T_{G_{k} / Q_{k}}(x, y) .
$$

Since $G_{k}-Q_{k}=G_{k+1}-e$ and $G_{k} / Q_{k}=G_{k+1} / e$, we have

$$
T_{G_{k}-Q_{k}}(x, y)=\left[x+g\left(y, p_{k+1}-1\right)-1\right] \text { and } T_{G_{k} / Q_{k}}(x, y)=y^{p_{k+1}-1},
$$

where $g(y, t)=y^{t-1}+y^{t-2}+\ldots+1$ for all integers $t \geq 1$. Thus

$$
T_{G}(x, y)=\left[x+g\left(y, p_{k+1}-1\right)-1\right] d_{k}(x, y)+y^{p_{k+1}-1} c_{k}(x, y) .
$$

We may use Lemma 8 to deduce:

$$
T_{G}(b, 0)=b d_{k}(b, 0)=b \prod_{j=0}^{k} g\left(b, s_{j}\right)
$$




$$
\begin{aligned}
T_{G}(0, b) & =\left[g\left(b, p_{k+1}-1\right)-1\right] d_{k}(0, b)+b^{p_{k+1}-1} c_{k}(0, b) \\
& \left.=\left[g\left(b, p_{k+1}-1\right)-1\right] \prod_{j=0}^{k} g\left(b, p_{j}\right)+b^{p_{k+1}-1} \prod_{j=0}^{k-1} g\left(b, p_{j}\right)\right] \\
& =\left[g\left(b, p_{k+1}\right)-1\right] \prod_{j=0}^{k} g\left(b, p_{j}\right)
\end{aligned}
$$

since $p_{k}=1$ (so $\left.g\left(b, p_{k}\right)=1\right)$. Similarly we have

$$
\begin{aligned}
T_{G}(a, a)= & {\left[a+g\left(a, p_{k+1}-1\right)-1\right] d_{k}(a, a)+a^{p_{k+1}-1} c_{k}(a, a) } \\
\leq & {\left[a+g\left(a, p_{k+1}-1\right)-1\right] \prod_{j=0}^{k} g\left(a, s_{j}\right) g\left(a, p_{j}\right)+} \\
& a^{p_{k+1}-1} \prod_{j=0}^{k-1} g\left(a, s_{j}\right) g\left(a, p_{j}\right) \\
\leq & {\left[a+g\left(a, p_{k+1}\right)-1\right] \prod_{j=0}^{k} g\left(a, s_{j}\right) g\left(a, p_{j}\right) }
\end{aligned}
$$

since $g\left(a, s_{k}\right) g\left(a, p_{k}\right) \geq 1$. We may use (2), (3), (4), and the facts that $g(b, t) \geq g(a, t)^{2}$ and $b[g(b, t+1)-1] \geq[a+g(a, t+1)-1]^{2}$ for all integers $t \geq 1$ (as was shown in the proofs of Lemma 9 and Claim 13) to deduce that $T_{G}(b, 0) T_{G}(0, b) \geq T_{G}(a, a)^{2}$.

\section{Closing Remarks}

(a) Conjecture 3 indicates that the hypothesis $b \geq a(a+2)$ of Theorem 4 may not be best possible. This hypothesis is needed in our proof to establish Claim 14. For example, suppose that $\left|P_{G}(e)\right|=2$ and that $\left(G_{0}, G_{1}\right)$ is the parallel/series reduction sequence of $G$. We have

$$
\begin{aligned}
T_{G}(x, y) & =d_{0}(x, y) T_{G_{0}-Q_{0}}(x, y)+c_{0}(x, y) T_{G_{0} / Q_{0}}(x, y) \\
& =T_{G_{0}-Q_{0}}(x, y)+(y+1) T_{G_{0} / Q_{0}}(x, y) .
\end{aligned}
$$

Thus

$$
\begin{aligned}
T_{G}(b, 0)+z_{0}^{2} T_{G}(0, b)= & T_{G_{0}-Q_{0}}(b, 0)+T_{G_{0} / Q_{0}}(b, 0)+ \\
& z_{0}^{2} T_{G_{0}-Q_{0}}(0, b)+z_{0}^{2}(b+1) T_{G_{0} / Q_{0}}(0, b)
\end{aligned}
$$




$$
\begin{aligned}
= & T_{G_{0}-Q_{0}}(b, 0)+z_{0}^{2} T_{G_{0}-Q_{0}}(b, 0)+ \\
& T_{G_{0} / Q_{0}}(b, 0)+z_{0}^{2}(b+1) T_{G_{0} / Q_{0}}(0, b) \\
\geq & 2 z_{0}\left[T_{G_{0}-Q_{0}}(a, a)+\sqrt{b+1} T_{G_{0} / Q_{0}}(a, a)\right]
\end{aligned}
$$

by Claim 11(b). On the other hand

$$
2 z_{0} T_{G}(a, a)=2 z_{0}\left[T_{G_{0}-Q_{0}}(a, a)+(a+1) T_{G_{0} / Q_{0}}(a, a)\right] .
$$

So, in order to deduce that $T_{G}(b, 0)+z_{0}^{2} T_{G}(0, b) \geq 2 z_{0} T_{G}(a, a)$, we need $\sqrt{b+1} \geq a+1$, that is to say $b \geq a(a+2)$.

(b) The proof of Theorem 4 is matroidal and can be used to obtain the following extension to matroids.

Theorem 15 Suppose $M$ is a matroid without loops and coloops, and $a \geq 0$, $b \geq a(a+2)$ are real numbers. Then

$$
T_{M}(b, 0) T_{M}(0, b) \geq T_{M}(a, a)^{2} .
$$

Indeed, the matroid proof would be in some sense simpler than the graph theoretic proof since we could make use of matroid duality (for example to deduce statements about series reduction from their dual statements about parallel reduction). I have chosen to give the graph theoretic proof to make it accessible to graph theorists unfamiliar with matroid theory.

(c) One may use the Cauchy-Schwarz inequality to deduce that, if $p(x, y)$ is a polynomial with nonnegative coefficients, then $p(b, c) p(c, b) \geq p(a, a)^{2}$ for all real numbers $a, b, c \geq 0$ with $b c \geq a^{2}$. The inequality for Tutte polynomials given in Theorem 4 does not hold for all 2-variable polynomials with nonnegative coefficients. For example, if $p(x, y)=x^{2}+8 x y+y^{2}, a=1$, and $b=3$, then $b \geq a(a+2)$ but $p(b, 0) p(0, b)<p(a, a)^{2}$.

Acknowledgement I would like to thank Mark Jerrum, Criel Merino, Robin Whitty for helpful discussions. I would also like to thank Gary Haggard, David Pearce and Gordan Royle for letting me use their software for computing Tutte polynomials.

\section{References}

[1] T. Brylawski and J. Oxley, The Tutte polynomial and its applications, in Matroid Applications, N. White, editor, Encyclopedia of Mathematics and its Applications Cambridge Univ. Press (1992) 123225. 
[2] R. Conde and C. Merino, A note on some inequalities for the Tutte polynomial of a matroid, preprint.

[3] C. Greene and T. Zaslavsky, On the interpretation of Whitney numbers through arrangements of hyperplanes, zonotopes, non-Radon partitions and orientations of graphs, Trans. Amer. Math. Soc. 280 (1983) $97-126$.

[4] M. Las Vergnas, Acyclic and totally cyclic orientations of combinatorial geometries, Discrete Math. 20 (1977) 51-61.

[5] C. Merino and D.J.A. Welsh, Forest colourings and acyclic orientations of the square lattice, Annals of Combinatorics 3 (1999) 417-429.

[6] R.P. Stanley, Acyclic orientations of graphs, Discrete Math. 5 (1973) 171-178.

[7] W. T. TutTe, A contribution to the theory of chromatic polynomials, Canad. J. Math. 6 (1953) 80-91.

[8] W. T. TutTe, Graph Theory, Cambridge University Press, Cambridge, 1984. 